Europe's Journal of Psychology, 6(3), pp. 149-173

www.ejop.org

\title{
The Impact of Humor in North American versus Middle East Cultures
}

\author{
Nicholas A. Kuiper \\ Univ ersity of Western Ontario
}

Shahe S. Kazarian

American University of Beirut

Jessica Sine

Univ ersity of Western Ontario

Margaret Bassil

American Univ ersity of Beirut

\section{Abstract}

North American (Canadian) and Middle East (Lebanese) participants rated their reactions to four different humorous comments (self-enhancing, affiliative, self-defeating, and aggressive), presented by others in brief scenarios. Consistent with predictions generated from a humor styles model originally formulated in a North American context, all participants responded most negatively to aggressive humorous comments by indicating the saddest mood, the highest ratings of rejection, and the least desire to continue interacting with the person making the comments. Only the North American participants showed a distinctive positive reaction to the self-enhancing humorous comments by displaying the happiest mood, the least rejection, and the greatest desire to continue with the interaction. In contrast, the Middle East Lebanese participants did not differentiate in their responses between self-enhancing, affiliative and self-defeating humorous comments. These findings were considered in light of cultural distinctions in collectivistic versus individualistic self-construals. Here, it was suggested that the collectivistic self-construals that characterize Lebanese Middle East participants may have blurred the self versus other distinctions in the humor styles model, thus leading to significantly less humor differentiation in a Lebanese Middle East context. The implications of these findings for further cross-cultural work on humor and its impact in social interactions was then considered.

Keywords: Humor, Cross-cultural, Lebanese, Self-construals, Social Interactions, Crosscultural, Canadian 
Considerable research has now documented the existence of several different humor styles. For example, one humor model proposed by Martin, Puhlik-Doris, Larsen, Gray, and Weir (2003) has suggested that certain humor styles may be quite adaptive for the individual, whereas other styles may be more maladaptive. In this approach, the two adaptive styles are affiliative and self-enhancing humor; whereas the two maladaptive styles are aggressive and self-defeating humor. Affiliative humor involves funny, non-hostile jokes, and spontaneous witty banter to amuse others in a respectful way. It is aimed at others and used in an adaptive manner to facilitate relationships and reduce interpersonal conflict. Aggressive humor, on the other hand, is intended to put others down by using sarcasm, teasing and ridicule. As such, the use of this maladaptive style may hurt or alienate others. In contrast, self-enhancing humor is often used as an adaptive coping mechanism, allowing the individual to adopt a humorous outlook on life and maintain a realistic perspective in stressful situations. Finally, self-defeating maladaptive humor involves selfdisparagement and allowing oneself to be the 'butt' of the joke, in order to gain the approval of others.

The four humor styles in the Martin et al. (2003) humor model are assessed via the Humor Styles Questionnaire (HSQ). A number of studies now provide evidence for the existence of these four styles across quite diverse cultures, including North American (Kuiper, Grimshaw, Leite \& Kirsh, 2004: Martin et al., 2003), Western European (Saraglou \& Scariot, 2002; Vernon, Martin, Schermer \& Mackie, 2008), Eastern (Chen \& Martin, 2007), and Middle East societies (Kalliny, Cruthirds \& Minor, 2006; Kazarian \& Martin, 2004; 2006; Taher, Kazarian \& Martin, 2008). In addition, these research studies has generally supported the distinction between adaptive and maladaptive humor styles, as higher levels of adaptive humor (either affiliative or self-enhancing) are usually associated with greater psychological well-being; whereas higher levels of maladaptive (e.g., self-defeating) humor are typically associated with increased depression and lower self-esteem. Moreover, these associations with well-being have also shown some degree of cross-cultural consistency, with the same general patterns often emerging in North American, Eastern and Middle East cultures.

Much less is known, however, about how the use of these humor styles may impact on another person in a typical social interaction. This issue was explored in the present set of studies by focusing on the responses made by individuals that were the recipients of humorous comments pertaining to each of the four humor styles. In this research, we were interested in determining the extent to which each type of humorous comment (affiliative, self-enhancing, aggressive, and self-defeating) might have either a positive or negative impact on the recipient's overall mood 
(happy-sad). We were also interested in determining whether the four types of humorous comments might have a differential effect on how much recipients felt accepted or rejected by the person making the comments; and the extent to which they would then want to continue interacting with that person. In addition to these perceptions that pertain more directly to social interactions, we also assessed the degree to which the various types of humorous comments might have a much broader impact on recipients by altering their cognitive apprais als of a stressful event they were dealing with. We examined cognitive appraisals, as prior research has shown that increased coping humor results in much greater flexibility when reassessing one's own stressful situations (Abel, 2002; Kuiper, Martin \& Olinger, 1993; Kuiper, McKenzie \& Belanger, 1995). As such, we were interested in determining whether similar re-appraisal effects might emerge after an individual is exposed to various types of humorous comments made by others.

Since relatively little cross-cultural research has investigated humor style effects from the perspective of the recipient, our first study offered an initial examination of this issue by using a Canadian North American sample. The remaining two studies then tested the extent to which the findings obtained in the first study also emerged in a Lebanese society. This Middle East culture was selected, as it is quite distinct from the individualistic North American culture that generated the original humor styles model. This cross-cultural issue is important to examine, as recent work by Taher et al. (2008) has found that the fit of the humor styles model to a Middle East collectivistic culture (Lebanese) was less well-defined than the fit typically found for individualistic cultures (e.g., North American, Western European). Although all four humor styles were still evident in the Taher et al., Lebanese sample, these styles were less distinct from one another than usual differences noted in North American samples (see also Kazarian \& Martin, 2004; 2006 for similar findings in further Lebanese samples). Furthermore, these investigators also found that the associations between the four humor styles and various indices of psychological well-being were less pronounced in the Lebanese samples, when compared to the same associations in more individualistic cultures. As such, Taher et al. (2008) have suggested that these findings are consistent with the proposal that there may be less differentiation among the four humor styles in collectivistic cultures, as these cultures do not display the same individualistic self-orientation underlying the original development of the humor styles model. These findings further suggest that the differential effects of the various humor styles on others may be less pronounced in collective cultures. This issue was examined in Studies 2 and 3, once we completed our exploration of the potential impact of humor styles in an individualistic North American Canadian sample. 


\section{Study 1: The Impact of Humorous Comments in a North American Canadian Culture}

In order to assess the responses to humorous comments made by others, participants were presented with two brief scenarios, each describing a moderately challenging situation faced by the participant. One scenario was academic, in which the participant was described as having done relatively poorly on an examination, and then going to meet with the teaching assistant (TA) to discuss the grade received. During this discussion, the TA responds with a humorous comment (either affiliative, self-enhancing, aggressive or self-defeating). Participants then rated how much this humorous comment impacted on their own mood (happy-sad), how much it made them feel accepted or rejected by the person making the humorous comment, and how much it made them want to continue interacting with this person. At a broader level, participants also indicated the degree to which the humorous comment made them feel more positive or negative about their own challenging situation (i.e., their low grade). In turn, the second scenario involved meeting with a casual friend to discuss the participant's recent breakup with a relatively long-term romantic partner. The causal friend then responds with a humorous comment (again pertaining to one of the four humor styles), followed by the same participant ratings as described above.

The humor styles model (Martin et al., 2003; Martin, 2007) was used to generate predictions for the expected pattern of findings. For the maladaptive humor styles, we hypothesized that the aggressive humor style comments would have the most negative impact on the recipient, resulting in the saddest mood, the highest feelings of rejection, and the lowest desire to continue the interaction. These predictions stem from the deliberately hurtful nature of aggressive humor that is directed towards the recipient (Martin et al., 2003; Martin, 2007). These characteristics of maladaptive humor would make the recipient want to withdraw from the situation, both emotionally and physically. At a broader level, these detrimental effects could then lead to an enhanced negative cognitive appraisal of the recipient's stressful event (i.e., low grades, relationship breakup).

In contrast to the above pattern for aggressive humor, we expected both of the adaptive humor comments to produce a significantly happier mood in the recipient, along with increased feelings of acceptance, and a greater desire to continue interacting. For affiliative humor, this pattern would reflect the basic facilitative nature of this humor style, which functions primarily to enhance social relationships (Martin, 2007). For the self-enhancing humorous comments, we expected a positive 
impact because of the moderately stressful events involved in our two scenarios. If recipients view the humorous self-enhancing comments by another person as an illustrative model of how to effectively cope with their own challenging events (low grade/relationship breakup), then recipients may experience much more positive mood and feel much more accepted by either the TA or casual friend. In turn, this modeling effect should greatly increase their desire to continue interacting with this individual, and may also help them cognitively re-appraise their own challenging event to be much more positive.

Predictions regarding the impact of self-defeating humor on recipients were less clear-cut. On the one hand, the humor styles model proposes that the function of self-defeating humor is to make the individual feel more accepted by other persons they interact with (Martin, 2007). In turn, this suggests that the use of self-defeating humor would be viewed by the recipients in a favorable manner, resulting in the recipients having a happier mood, greater feelings of acceptance, and an increased desire to continue interacting with the individual using self-defeating humor. This could also lead to more positive cognitive appraisals and higher ratings of similarity to the recipient's own response in the same type of situation. On the other hand, the explicit demeaning and ingratiating nature of self-defeating humor may result in a negative distancing response by recipients. This distancing reaction would be evident in less positive mood, greater feelings of rejection, and reduced desire to interact with the individual using this adverse humor style. Furthermore, this distancing effect may also impact more broadly by making the cognitive reappraisals of the stressful situations (low grade, relationship difficulties) much more negative.

\section{Method}

\section{Participants}

A sample of 173 university students (139 females, 34 males) enrolled in introductory psychology courses at a large English-speaking North American (Canadian) university participated in this study, in partial fulfillment of course requirements. Their mean age was 18.79 (SD =1.68), with a range from 17 to 33 .

Materials

Two different stressful event descriptions were used. In the academic scenario, participants read the following: 
Imagine that you are going to your TA's office to get your grade back from an exam you wrote two weeks ago. This is an important course for you, and you studied hard for the exam. You had expected that you would do well, but when you get your exam back, your grade is well below your usual average. You also find out that although you did pass the exam, your grade is below the class average.

In the interpersonal scenario the following information was presented:

Imagine that you are in the university cafeteria having a snack with a casual friend from one of your classes. You see this friend about once a week outside of class and spend some time discussing various personal events. Today you tell your casual friend that the person you have been dating for the past year is now seriously considering breaking off the relationship.

Directly beneath each scenario, each participant was presented with a description of one of four possible humorous comments (affiliative, self-enhancing, aggressive, or self-defeating), made by either the TA (in the academic scenario) or the casual friend (in the interpersonal scenario), in response to the stressful event described in the relevant scenario. The four different types of humorous comments are presented below.

Affiliative Humor Response: Your TA [casual friend] responds by saying funny things that do not focus on your exam performance [dating relationship problems]. The TA [casual friend] provides some spontaneous witty banter and then tells a few non-hostile jokes to amuse you, and help put you at ease. Your TA's [casual friend's] use of this tolerant humor indicates that your TA [casual friend] is appropriately respectful of self and others, and does not take things overly seriously.

Self-Enhancing Humor Response: Your TA [casual friend] responds by saying funny things about a time when they had performed poorly on an exam [had difficulties in a dating relationship]. The TA [casual friend] comments about how they used humor to help maintain a realistic perspective when faced with this upsetting event. Your TA's [casual friend's] use of this coping humor indicates that your TA [casual friend] has a generally humorous, but still realistic outlook on life, and is frequently amused by the incongruities of everyday events.

Aggressive Humor Response: Your TA [casual friend] responds by saying 
funny things about your exam performance [dating relationship problems], but things that are sarcastic and critical of you. The TA [casual friend] provides humorous comments that ridicule your performance and ability. Your TA's [casual friend's] use of this putdown humor indicates that your TA [casual friend] often expresses humor without consideration of its potential impact to be hurtful and alienate others.

Self-defeating Humor Response: Your TA [casual friend] responds by saying funny things about a time when they had performed poorly on an exam [had difficulties in a dating relationship]. The TA [casual friend] comments about how they made several jokes about their own intellectual faults and academic weaknesses [their own deficits in dating skills and interpersonal weaknesses], in order to let others laugh at their expense. Your TA's [casual friend's] use of this self-disparaging humor indicates that your TA [casual friend] often allows themselves to be the "butt" of jokes, and will laugh along when ridiculed by others.

Imme diately beneath the humorous response were several questions that were each rated on a 5-point scale. The first asked how the humorous comment of the other individual (TA or casual friend) made the recipient (i.e., participant) feel in this situation, ranging from happy (1) to sad (5). The next question asked how much this humorous comment would make the recipient feel either accepted (1) or rejected (5). Following this, participants then indicated how much they would want to continue interacting with the TA or casual friend that used this type of humor, with responses ranging from (1) "not at all" to (5) "very much." Finally, cognitive reappraisals of the stressful event being discussed in the scenario (poor exam performance or dating problems) were assessed on a 5 point scale ranging from (1) "much more negative" to (5) "much more positive."

\section{Procedure}

Participants were tested in small groups of up to 15. After completing an informed consent form, each participant received a booklet. Four booklets were constructed, which corresponded to the four different types of humorous comments (selfenhancing, affiliative, self-defeating, and aggressive). Within a booklet, participants were presented with both scenarios (academic and interpersonal), but for only one of the four types of humorous comments. The presentation order of scenarios was varied across booklets. Within each session, participants were randomly assigned to one of the four humorous comment conditions. Upon completion of the booklet, 
participants received a debriefing form that offered further information regarding the study.

\section{Results and Discussion}

Each rating was analyzed using a $4 \times 2$ (Humorous Comments $\times$ Scenario) analysis of variance (ANOVA). For each analysis, the between-subjects factor was humorous comments (self-enhancing, affiliative, self-defeating, and aggressive); whereas the repeated measures factor was scenario (academic, interpersonal). All significant ANOVA effects were followed up, when required, with t-tests on the appropriate cell means.

Happy-Sad Mood. A significant main effect was found for humorous comments, $F=$ 17.42, $p<.001$, with the means and standard deviations for this North American Canadian sample shown in the top row of Table 1. As expected, recipients of aggressive humorous comments felt the saddest, compared to any of the remaining humorous comments, all $\mathrm{p}$ 's <.001. In contrast, recipients of self-enhancing humorous comments displayed the happiest mood, compared to individuals receiving either aggressive or self-defeating humorous comments, both $p$ 's <.001. Furthermore, these mood ratings for self-enhancing comments were even happier than the same ratings associated with the affiliative humorous comments, $p<.05$. The affiliative humorous comments, however, did not show any difference in mood, when compared to the self-defeating humorous comments. Finally, the ANOVA revealed that the only remaining significant source of variance was a main effect for scenario, $F=4.31, p<.05$. Here, individuals were less happy overall in the academic than interpersonal scenario (respective means of 3.24 versus 3.05).

Accepted-Rejected. The ANOVA for this measure revealed a significant main effect for humorous comments, $F=29.72, p<.001$. As shown in Table 1, Canadian participants felt the most accepted after the self-enhancing humorous comment and felt the most rejected after the aggressive humorous comment, compared with any of the remaining comments, all $p$ 's $<.001$. As was the case for the happy-sad mood rating reported directly above, no significant difference was found between the affiliative and self-defeating humorous comments for these ratings. 
Table 1: Study 1 North Americ an Canadian Sample - Means and SDs

Humorous Comments

Self-Enhancing Affiliative Self-Defeating Aggressive

Recipients' Ratings

$\begin{array}{lccccc}\text { Happy-Sad Mood } & M & 2.61 & 3.00 & 3.15 & 3.79 \\ & S D & 0.93 & 0.97 & 1.01 & 1.17 \\ & & & & & \\ \text { Accepted-Rejected } & M & 2.24 & 2.94 & 2.60 & 3.78 \\ & S D & 0.85 & 1.09 & 0.95 & 1.02 \\ \text { Continue Interaction } & M & 3.81 & 3.03 & 3.34 & 1.90 \\ & S D & 1.05 & 1.32 & 1.15 & 0.99 \\ & & & & & \\ \text { Cognitive Re-apprais al M } & 3.55 & 3.02 & 3.05 & 1.94 \\ & S D & 0.74 & 0.76 & 0.88 & 0.83\end{array}$

Notes. $\mathrm{n}=173$ All ratings were made on 5 point scales. For Happy-Sad, higher numbers are sadder; For Accept-Reject, higher numbers are more rejected; For Continue Interaction, higher numbers are more desire to interact; For Cognitive Re-appraisal, higher numbers are much more positive.

Desire to Continue Interaction. The ANOVA indicated that only the main effect of humorous comments was significant, $F=31.97, p<.001$. As shown in Table 1, an aggressive humorous comment significantly decreased the desire to continue the interaction, compared to each remaining type of humorous comment, all $p$ 's $<.001$. In contrast, these North American participants felt the greatest desire to continue the interaction after receiving a self-enhancing humorous comment, compared to each of the remaining humorous comments, all $p$ 's <.01. Once again, there was no significant difference between the affiliative and self-defeating humorous comments, indic ating equivalent desire to continue interacting with the TA or casual friend.

Cognitive Re-appraisals. The significant main effect of scenario, $F=4.95, p<.05$, indicated that individuals were generally more positive in their cognitive re- 
appraisals of the interpersonal event than the academic event (respective means of 2.96 versus 2.80). More importantly, the ANOVA also revealed a significant main effect for humorous comments, $F=47.44, p<.001$. As shown in Table 1, North American participants re-appraised their stressful events (poor exam performance and relationship problems) more negatively after the TA or casual friend responded with an aggressive humorous comment, compared to each of the remaining humorous comments, all $p$ 's $<.001$. Conversely, participants had the most positive cognitive re-appraisals of their stressful events after receiving a self-enhancing humorous comment, compared to each of the other humorous comments, all $p$ 's < .001 . Finally, there was once again no difference between the affiliative and selfdefeating humorous comments. In other words, cognitive re-appraisals were equiv alent following either of these two types of humorous comments.

Summary and Conclusions. The findings from Study 1 provided clear initial evidence that humorous comments can have a pronounced impact on recipients, and that this impact can vary from very positive to quite ne gative. Consistent with predictions generated from the humor styles model (Martin et al., 2003; Martin, 2007) we found that for both types of situations (academic and interpersonal) aggressive humorous comments have the most detrimental impact on recipients, resulting in the saddest mood, highest feelings of rejection, and the lowest desire to continue interacting. These aggressive humorous comments also had a broad impact on the recipient, as they lead to the most negative cognitive appraisals of the recipients' stressful events (i.e., low grades and relationship breakups). In further accord with the humor styles model, we found that humorous comments pertaining to both adaptive humor styles (affiliative and self-enhancing) resulted in a much happier mood, greater acceptance, and a greater willingness to continue interacting. These positive effects also extended to include much more positive cognitive appraisals of the stressful situations being discussed. Interestingly, all of these effects were significantly more positive for the self-enhancing humorous condition, suggesting that these particular comments provided a strong modeling example for recipients to use when dealing with their own stressful circumstances. Finally, it should be noted that the findings for the self-defeating humorous comments were less negative than those for aggressive comments, but were also less positive than those for self-enhancing comments. This pattern suggests that the impact of self-defeating humorous comments cannot be considered either highly maladaptive or adaptive, but rather leads to more ambivalent views on the part of the recipient. 


\section{Study 2: The Impact of Humorous Comments in a Middle East Culture}

Using a North American Canadian sample, Study 1 findings clearly indicated that various types of humorous comments can have a strong and differential impact on recipients. It is not yet known, however, whether these humor effects represent cultural universals that are broadly evident across different cultures; or rather are culture-bound and specific to the same individualistic culture that originally generated the humor styles model. To address this issue, we conducted a second study using the same procedures as Study 1, but using a Lebanese group. This sample was selected because of the strong collectivist underpinnings that mark this Middle East culture (Kazarian, 2005; Kazarian; in press). In this culture, the self is generally construed as being interdependent, with an emphasis on connectedness with others, group cohesion, harmony, and cooperation (Dwairy, Achaoui, Abouserie \& Farah, 2006). This collectivist focus is in distinct contrast to the independent, unique and autonomous self-construals that generally characterize individualistic societies, such as those found in North America and Western Europe (Markus \& Kitayama, 1991; Singelis, 1994).

This distinction in self-construals between individualistic and collective cultures is important, as the conceptual development of the humor styles model derived from self-construals that stressed independence and autonomy, rather than group cohesion and cooperation (Taher et al., 2008). Thus, in a collective culture, the self versus other focus of the various humor styles (e.g., affiliative is other directed; selfenhancing is self-directed) becomes much more blurred and less distinct than in an individualistic culture. As such, we expected that the Lebanese participants in this study would not distinguish as clearly between the humor styles, thus showing less differentiation between the various humorous comments in terms of their effects. such a pattern would support the proposal that certain aspects of the humor styles model are more cultural-bound, as they are sensitive to cultural variations in selfconstruals. The opposing position, of course, is that the pattern of findings for our Lebanese participants would be identical to that obtained for the North American Canadian sample, thus supporting a cultural universal interpretation.

\section{Method}

\section{Participants}

Participants were 198 undergraduate students (114 females, 84 males) from the American University of Beirut, a private coeducational institution in which English is 
the language of instruction. In terms of nationality, $69.7 \%$ of the sample was Lebanese, $25.6 \%$ were Lebanese with dual nationality, and $4.6 \%$ were other (e.g., Palestinian born in Lebanon). The mean age of this sample was 19.30, with a standard deviation of 1.27 years.

\section{Materials and Procedure}

The same materials used in Study 1 were also used in this study. A minor change was made to the last sentence for the interpersonal scenario. Here, the specific interpersonal stressful event now being discussed was changed from dating concerns to family difficulties, in order to better reflect the central social role of the family in Lebanese culture (Kazarian, 2005). As such, this sentence now read "Today you tell your casual friend that you are having difficulties with your parents who seem very unhappy about your going out with friends and staying late at night." All the remaining aspects of this study, including materials and procedure, were identical to Study 1. Thus, each participant read two scenarios (academic and interpersonal), that were each followed by one of the four humorous types of comments. The order of scenarios was again counterbalanced across participants. The questions and rating scales that followed the scenarios were identical to Study 1, except for some very minor wording changes to accommodate the different stressful event now being discussed in the interpersonal scenario. All participants completed a consent form prior to receiving the materials for this study, and were given a debriefing form at the end of the study.

\section{Results and Discussion}

A series of $4 \times 2$ ANOVAs (Humorous Comments $\times$ Scenario) were used to analyze the set of dependent measures for this study, with follow-up t-tests on appropriate cell means, when required.

Happy-Sad Mood. The ANOVA revealed that main effect for humorous comments was significant, $F=9.35, p<.01$. The means and standard deviations for this sole significant effect are shown in the top row of Table 2. Consistent with the North American Canadian sample in Study 1, the Lebanese participants in this study were saddest after receiving aggressive humorous comments, compared to any of the remaining types of humorous comments, all p's <.001. In contrast to the North American sample, however, there were no further significant differences in mood between affiliative, self-enhancing, and self-defeating humorous comments. As such, this pattern clearly indicates less differentiation among the humor styles 
comments for these Lebanese participants, in terms of subsequent impact on mood. In particular, self-enhancing, affiliative and self-defeating humorous comments all resulted in the same level of mood; with all three leading to significantly less sad mood than shown following aggressive humor comments.

Table 2: Study 2 Lebanese Sample (English-speaking) - Means and SDs

Humorous Comments

Self-Enhancing Affiliative Self-Defeating Aggressive

Recipients' Ratings

$\begin{array}{lrrrrr}\text { Happy-Sad Mood } & M & 2.88 & 2.94 & 2.93 & 3.61 \\ & \text { SD } & 0.60 & 0.82 & 0.63 & 0.74 \\ & & & & & \\ \text { Accepted-Rejected } & M & 2.56 & 2.88 & 2.63 & 3.25 \\ & S D & 0.52 & 0.76 & 0.62 & 0.92 \\ \text { Continue Interaction } & M & 3.66 & 3.01 & 3.22 & 2.13 \\ & S D & 0.79 & 0.96 & 0.84 & 0.87 \\ & & & & & \\ \text { Cognitive Reapprais al } & M & 3.37 & 2.92 & 3.25 & 2.37 \\ & S D & 0.62 & 0.74 & 0.54 & 0.64\end{array}$

Notes. $\mathrm{n}=198$ All ratings were made on 5 point scales. For Happy-Sad, higher numbers are sadder; For Accept-Reject, higher numbers are more rejected; For Continue Interaction, higher numbers are more desire to interact; For Cognitive Re-appraisal, higher numbers are much more positive.

Accepted-Rejected. The means and standard deviations for this measure are also shown in Table 2, with the ANOVA revealing a signific ant main effect for humorous comments, $F=9.05, p<.01$. Consistent with the North American sample, the Lebanese participants in this study felt the most rejected after the aggressive humorous comment, compared with any of the remaining comments, all $p$ 's <.01. Contrary to the North American sample, however, the Lebanese participants did not feel significantly more accepted after self-enhancing comments. Instead, all three remaining styles of humorous comments (affiliative, self-enhancing, and self- 
defeating) resulted in equivalent ratings of being accepted-rejected. Once again, this pattern points to much less differentiation among these three humor style comments for these Middle East participants.

Desire to Continue Interaction. The ANOVA indicated that only the main effect of humorous comments was significant, $F=27.05, p<.001$. Table 2 shows that Lebanese participants felt the greatest desire to continue the interaction after receiving a self-enhancing humorous comment, compared to either the aggressive or affiliative humorous comments, both $p$ 's $<.01$. This finding was very similar to that displayed for the North American sample. However, the comparisons between both self-enhancing and self-defeating humorous comments, and aggressive and selfdefeating humorous comments, were no longer significant for the Lebanese participants, highlighting signific antly less differentiation among these humor styles for this group.

Cognitive Re-appraisals. Humorous comments were the only significant source of variance in this ANOVA, $F=24.10, P<.001$. As shown in Table 2, Lebanese participants re-appraised their stressful events (poor exam performance and family problems) more negatively after an aggressive humorous comment, compared to all three of the remaining humorous comments, all $p$ 's $<.001$. This pattern was identical to that displayed by the North American participants in Study 1. Also congruent with the Canadian sample, Lebanese participants displayed the most positive cognitive re-appraisals after receiving a self-enhancing humorous comment, compared with either affiliative or aggressive humorous comments, all p's <.001. In further accord with the North American sample, the current participants also did not display a significant difference in cognitive re-appraisals between an affiliative and self-defeating humorous comment. Overall, this pattern indicates that the Lebanese sample showed the same degree of differentiation among the humor styles as the North American Canadian sample, in terms of impact on cognitive re-appraisals.

Summary and Conclusions. The results for this Lebanese sample revealed both similarities and differences, when compared with the North American findings for Study 1. In particular, both cultures rated aggressive humorous comments as being the most negative of all, resulting in the saddest mood, the highest feelings of rejection, and the lowest desire to continue interacting. Both cultures also showed very similar patterns for the cognitive re-appraisals, with aggressive comments again resulting in the most negative appraisals. Overall, these findings suggest that the impact of aggressive humor appears to be universal and broad across these two cultures. 
Contrary to the North American sample, however, the Lebanese participants did not distinguish among self-enhancing, affiliative and self-defeating humorous comments when rating subsequent impact on happy-sad mood, feeling accepted-rejected, or wanting to continue the interaction. This pattern suggests that this Middle East culture does not differentiate among these particular humor styles when considering their impact, and is thus consistent with a North American culture-bound interpretation for these specific effects of humorous comments.

\section{Study 3: A Further Test of the Impact of Humorous Comments in a Middle East Lebanese Culture}

Thus far, the findings from Studies 1 and 2 suggest that some of the obtained effects for the humor style comments appear to be cultural universals, whereas others appear to be culture-bound. However, one possible concern in Study 2 is that we used an English-speaking sample of Lebanese participants. Although the continued administration of all testing materials in English allowed us to closely duplicate the procedures used in Study 1, it did not allow us to examine the potential impact of humorous comments that are made and received in the native Arabic language. Taher et al. (2008) have indicated that it is very important to do so, not only from the theoretical perspective of distinct underlying self-construals (i.e., collective versus individualistic), but also from a measurement perspective.

Accordingly, our third and final study was conducted entirely in the Arabic language, using a further Lebanese sample. In this study, we were once again interested in exploring the extent to which the humorous effects documented previously in Studies 1 and 2 could be viewed as being either cultural universals, or being culture-bound to the individualistic North American society that originally developed the humor styles model. In accord with the proposal that collective selfconstruals may be even more germane in this Arabic-speaking sample (Taher et al., 2008), we thus expected to see, at a minimum, the same level of culture-bound effects in Study 3 as observed in Study 2. Furthermore, it remained possible that these culture-bound effects would extend more broadly to include cognitive appraisals. If so, this would also highlight the importance of including native language assessments in any research examining cultural similarities or differences. 
Method

Participants

Participants were 243 undergraduate students (120 females, 123 males) from the American University of Beirut and the Lebanese University, the largest state-run institution in which Arabic is the primary language of instruction. In terms of nationality, $77.8 \%$ of the sample was Lebanese, $13.7 \%$ were Lebanese with dual nationality, and $8.5 \%$ were other. The mean age of this sample was 20.1 , with a standard deviation of 3.50 ye ars.

Materials and Procedure

The materials and procedure used in Study 3 were identical to those used in Study 2 , except that all of the questionnaires were now presented in Arabic, rather than in English.

\section{Results and Discussion}

A $4 \times 2$ ANOVA (Humorous Comments $\times$ Scenario) was used to analyze each dependent measure in this study, with follow-up t-tests on appropriate cell means, when required.

Happy-Sad Mood. The top row of Table 3 shows the happy-sad cell means and standard deviations associated with the sole significant main effect of humorous comments, $F=17.38, p<.001$. Consistent with both the Canadian and Englishspeaking Lebanese samples, the Arabic-speaking Lebanese participants in this study were also saddest after aggressive humorous comments, compared to each of the other types of humorous comments, all $p$ 's < .001. In accord with the Englishspeaking Lebanese sample, the Arabic-speaking Lebanese participants also did not show any significant differences in happy-sad mood between self-enhancing, affiliative, and self-defeating humorous comments. This pattern was distinct from the Canadian group, in which self-enhancing comments resulted in significantly happier ratings than either affiliative or self-defeating humorous comments. As such, these findings highlight a reduced degree of differentiation for both of the Lebanese groups. In particular, the Lebanese participants, regardless of whether they were English or Arabic-speaking, did not distinguish between self-enhancing, affiliative or self-defeating humor. It was only aggressive humor that showed a distinct impact on sad mood for these participants. 
Table 3: Study 3 Lebanese Sample (Arabic-speaking)- Means and SDs

Humorous Comments

Self-Enhancing Affiliative Self-Defeating Aggressive

Recipients' Ratings

$\begin{array}{lccccc}\text { Happy-Sad Mood } & M & 2.72 & 2.82 & 2.82 & 3.74 \\ & S D & 0.92 & 0.85 & 0.84 & 0.79 \\ & & & & & \\ \text { Accepted-Rejected } & M & 2.78 & 2.88 & 2.85 & 3.71 \\ & S D & 0.86 & 0.96 & 0.86 & 0.95 \\ \text { Continue Interaction } & M & 3.72 & 3.18 & 3.23 & 1.92 \\ & S D & 0.65 & 0.91 & 0.85 & 0.69 \\ & & & & & \\ \text { Cognitive Reapprais al } & M & 3.32 & 3.00 & 3.95 & 2.58 \\ & S D & 0.67 & 0.69 & 0.80 & 0.71\end{array}$

Notes. $\quad n=243$ All ratings were made on 5 point scales. For Happy-Sad, higher numbers are sadder; For Accept-Reject, higher numbers are more rejected; For Continue Interaction, higher numbers are more desire to interact; For Cognitive Re-appraisal, higher numbers are much more positive

Accepted-Rejected. The ANOVA revealed a sole significant main effect for humorous comments, $F=12.97, p<.01$. Consistent with the findings for both prior samples, Arabic-speaking Lebanese participants also felt the most rejected after the aggressive humorous comment, compared to any of the other humorous comments, all $p$ 's <.001 (see Table 3). Consistent with the English-speaking Lebanese sample, all three of the remaining types of humorous comments (affiliative, self-enhancing, and self-defeating) resulted in equivalent levels of feeling accepted-rejected. This pattern for both Lebanese samples is distinct from the North American Canadian sample, in which participants felt significantly more accepted after self-enhancing comments. Furthermore, it indicates no differentiation between self-enhancing, affiliative and self-defeating humor effects for all Lebanese participants (either English or Arabic-speaking). 
Desire to Continue Interaction. Only the main effect of humorous comments was significant, $F=9.74, p<.01$. The cell means and standard deviations for this effect are shown in Table 3. Consistent with both the Canadian and English-speaking Lebanese samples, the least desire to continue the interaction $w$ as after an aggressive humorous comment, compared with any of the other humorous comments, all p's < .01. Distinct from the English-speaking Lebanese participants, the participants in this study no longer differentiated between self-enhancing and affiliative humor. In other words, the Arabic-speaking Lebanese group showed even less differentiation than the English-speaking Lebanese group, as the former group did not distinguish between any of the three humor styles (self-enhancing, affiliative, self-defeating) when indicating their desire to continue interacting.

Cognitive Re-appraisals. The ANOVA revealed that there were no significant sources of variance for cognitive re-appraisals. Thus, contrary to the signific ant effects found in both Studies 1 and 2 for this measure, the present study found that Arabicspeaking Lebanese participants did not show any cognitive re-appraisal distinctions across the four types of humorous comments. Thus, in marked contrast to the pattern displayed by both the Canadian and English-speaking Lebanese samples (i.e., more positive re-appraisals after self-enhancing comments and more negative re-apprais als after aggressive comments), the Arabic-speaking Lebanese sample did not vary their cognitive re-appraisals after different types of humorous comments. This pattern suggests that the Arabic-speaking Lebanese group did not differentiate among any of the four humor styles, in terms of their subsequent impact on cognitive re-apprais als.

Summary and Conclusions. The findings from this third study both reinforce and extend the conclusions drawn from the previous two studies. To begin, the effects of aggressive humorous comments were strikingly consistent across all three samples, suggesting a strong universal component for the impact of these comments. In particular, aggressive humorous comments have a consistent detrimental impact on others, inducing negative mood and rejection, followed by a desire to limit interactions. In contrast, the effects of self-enhancing humorous comments are culture-bound to the North Americ an Canadian sample, as neither Lebanese group (English or Arabic speaking) distinguished these comments from either affiliative or self-defeating comments. Furthermore, it also appears that the impact of humorous comments on cognitive re-appraisals is limited to both English-speaking samples, as the Arabic speaking Lebanese participants did not differentiate between any of the types of humorous comments when providing cognitive re-appraisals. 


\section{General Discussion}

The main purpose of the present research was to investigate the extent to which the impact of the four humor styles identified by Martin et al. (2003) may be similar or distinct across various cultures. In order to do so, however, we first had to deter mine whether the humorous comments pertaining to each humor style actually did have an effect on recipients. This was necessary, as the majority of research thus far has focused primarily on identifying the existence of the four humor styles across various cultures, and then determining how these humor styles may relate to other personality characteristics and psychological well-being (Chen \& Martin, 2007; Kazarian \& Martin, 2006; Kuiper, et al., 2004; Martin, et al., 2003; Martin, 2007; Saroglou \& Scariot, 2002). In contrast, extremely little research has explored the potential impact of using these humor styles on others, particularly from a crosscultural perspective.

As such, our first study examined this issue in a North American individualistic culture. The findings from this Canadian study provided strong initial support for the impact of humor styles on others, as certain humorous comments did have significant and differential effects on the recipients. Consistent with predictions generated from the humor styles model, we found that aggressive humorous comments had a powerful negative effect on recipients, resulting in the saddest mood, the greatest feelings of rejection, and the lowest desire to continue the interaction. These comments also caused the recipients to cognitively re-appraise their own stressful event (i.e., poor exam performance, relationship problems) in an even more negative manner. This pattern of findings is congruent with the humor styles model, since aggressive humor involves ridiculing and alienating other people (Martin et al., 2003). As such, it was expected that these aggressive humorous comments would have a harmful personal impact on how an individual feels and reacts to various social interactions (e.g., ac ademic, interpersonal).

Conversely, and also as predicted, the self-enhancing humorous comments had precisely the opposite impact, resulting in the happiest mood, greatest feelings of acceptance, and the most desire to continue interacting. After receiving these humorous comments, recipients also reacted with the most positive change in the cognitive re-appraisals of their own stressful events. As such, these findings from Study 1 offer strong initial support for the impact of humor on others, not only for constructs relating directly to social interactions (e.g., one's own mood, feeling accepted or rejected, continuing the interaction); but also more broadly for constructs relating to cognitive re-appraisals of ongoing stressful events. 
The extensive breadth of these humor effects in Study 1 is consistent with the often stated adage that humor can function as a powerful social interaction tool (Martin, 2007). Although prior work has demonstrated that greater coping humor is linked to more positive cognitive appraisals of one's own stressful events (Abel, 2002; Kuiper et al., 1993; Kuiper et al., 1995), the present work provides the first demonstration that humor use can also alter the cognitive re-appraisals that are made by others. Furthermore, the present work is the first demonstration that these other-referent effects of humor on cognitive re-appraisals can be either positive or negative, depending upon the type of humorous comments being made (i.e., self-enhancing versus aggressive). As such, this pattern provides further strong empirical support for the adaptive versus maladaptive distinction in the Martin et al. (2003) humor styles model, as well as the pervasive power of humor use in an interpersonal context.

It is of further interest to note that in Study 1 neither self-defeating nor affiliative humorous comments had a very strong negative or positive impact on others, when compared to the more pronounced effects of aggressive and self-enhancing humorous comments. Although self-defeating humor has clearly documented detrimental effects on the well-being of the user (e.g., Kuiper et al., 2004), the present findings indicate that the use of this humor style does not inflict strong negative feelings in others, nor impede social relationships. Thus, self-defeating humor use does not appear to be as maladaptive as aggressive humor. In fact, the present findings suggest that the use of self-defeating humor is tolerated to the same extent as the use of affiliative humorous comments, with both of these comments yielding greater acceptance on the part of others than aggressive humor. This pattern of findings suggests that the adaptive versus maladaptive distinctions in the humor styles model may sometimes overlap to a considerable degree, with further refinements of the model being required.

Following our initial look at the effects of humor within a North American Canadian sample, we then directed our attention towards cross-cultural issues in the use of humor and its impact on recipients in a collectivistic Middle East culture. In this regard, Study 2 employed an English-speaking Lebanese sample; whereas Study 3 employed an Arabic-speaking Lebanese sample. The results from these two studies were then compared and contrasted with the findings from our initial Canadian sample, in order to determine which humor use findings may be cultural universals and which may be culture-bound. Across the three studies evidence was obtained for both. 
To begin, the use of aggressive humorous comments appears to have culturally universal effects on recipients, as the results for this particular humor style were highly consistent across all three studies. In all cases, recipients of this type of humorous comments showed the most detrimental effects, including the saddest mood, the highest degree of perceived rejection, the least desire to continue the interaction and the most negative change in cognitive re-appraisals for their own stressful events. It is clear from these findings that using aggressive humorous comments is highly detrimental to recipients, regardless of whether the culture involved is individualistic or collectivistic; and regardless of whether the use of humor is in the English language or in Arabic.

Other findings, however, point to humor use effects which are much more specific to the North American individualistic culture from which the humor styles model originated. These culture-bound findings relate to the use of self-enhancing humorous comments, which primarily have a positive impact for recipients in a North American culture. When turning to a more collectivist culture, these positive effects of using self-enhancing humorous comments dissipate. In Study 2, for example, the English-speaking Lebanese recipients of self-enhancing humor no longer displayed a happier mood, or perceived a higher degree of acceptance than recipients of either affiliative or self-defeating humorous comments. This same reduced differentiation was also evident in the Arabic-speaking Lebanese sample of Study 3. Thus, as predicted by Taher et al.'s (2008) notion of more blurred self-other distinctions for collectivistic cultures, the findings from our studies point to significantly less differentiation among these three humor styles for both English and Arabicspeaking Lebanese participants, when compared to the North American Canadian participants.

Interestingly, several additional findings indicated that the Arabic-speaking sample showed even less differentiation across the humor styles than the English-speaking Lebanese group. As one illustration, the Arabic-speaking participants did not differentiate between self-enhancing, affiliative, or self-defeating humorous comments, when indicating their desire to continue interacting. Furthermore, this group did not distinguish between any of the four humor styles when making cognitive re-appraisals of their own stressful situations (i.e., low grade, poor relationship with parents), suggesting that the breadth of humor effect found earlier is not evident in this group. Thus, consistent with Taher et al. (2008), our pattern of findings clearly indicates that the differential effects of the humor styles on others are even more limited in a collectivistic group tested in their own native language (in this case, Arabic), rather than in English. As such, these language-specific findings 
suggest that some degree of caution should be exercised regarding the conclusions drawn from cross-cultural research studies which do not involve native language participation.

More generally, the present findings suggest that a great deal of care needs to be exercised when applying the Martin et al. (2003) humor styles model to cultures that differ significantly from the North American individualistic culture that originally informed the development of this model. Considerable additional research is required to more firmly delineate the boundary conditions that may be associated with the effects of this humor model, when applied cross-culturally. One avenue of research, for example, might investigate the extent to which similar collectivistic effects for humor might be noted in a Chinese culture (Chen \& Martin, 2007). It would also be important for further research to move beyond the vignette approach adopted in the present set of studies. Although humor findings based upon this procedure can be quite informative (e.g., Butzer \& Kuiper, 2008), it is also important to examine humor effects using other research paradigms, including direct observation techniques (Campbell, Martin \& Ward, 2008). This work should also move beyond the university student samples we employed to include more community-based samples (Taher et al., 2008). Finally, it is also important that future cross-cultural work provides a direct assessment of the underlying self-construals that are thought to interact with the impact of humor. Although past work has generally supported the notion that a Middle East culture is much more aligned with collectivistic self-construals (Kazarian, 2005), it should be noted that recent crosscultural research stresses the importance of measuring these construals more directly (Harb \& Smith, 2008; Kolstad \& Horpestad, 2009). For now, however, our findings provide a starting point for exploring the extent to which these four humor styles may play a differential role in social interactions and interpersonal relationships across various cultures.

Acknowledgements. We would like to thank Yasmine Nassif for her involvement in the Arabic translation of the scenarios used in this research and May Awaida for her assistance in the data gathering process in Lebanon.

\section{References}

Abel, M. H. (2002). Humor, stress, and coping strategies. Humor: International Journal of Humor Research, 15, 365-381. 
Butzer, B., \& Kuiper, N. A. (2008). Humor use in romantic relationships: The effects of relationship satisfaction and pleasant versus conflict situations. Journal of Psychology: Interdisciplinary and Applied, 142, 245-260.

Campbell, L., Martin, R.A., \& Ward, J.R. (2008). An observational study of humor use during a conflict discussion. Personal Relationships, 15, 41-55.

Chen, G-H., \& Martin, R. A. (2007). A comparison of humor styles, coping humor, and mental health between Chinese and Canadian university students. Humor: International Journal of Humor Research, 20, 215-234.

Dwairy, M., Achaoui, M., Abouserie, R., \& Farah, R. (2006). Adolescent-family connectedness among Arabs: A second cross-regional research study. Journal of CrossCultural Psychology, 37, 248-261.

Harb, C., \& Smith, P. (2008). Self-construals across cultures: Beyond IndependenceInterdependence. Journal of Cross-cultural Psychology, 39(2), 178-197.

Kalliny, M., Cruthirds, K.W., \& Minor, M.S. (2006). Differences between American, Egyptian and Lebanese Humor Styles: Implications for international management. International Journal of Cross Cultural Management, 6, 121-134.

Kazarian, S.S. (2005). Family functioning, cultural orientation, and psychological wellbeing among university students in Lebanon. Journal of Social Psychology, 145, 141-152.

Kazarian, S. S. (in press). Humor in the collectivist Arab Middle East: The case of Lebanon. Humor: International Journal of Humor Research.

Kazarian, S. \& Martin, R. (2004). Humor styles, personality and well-being among Lebanese university students. European Journal of Personality, 18, 209-219.

Kazarian, S.S., \& Martin, R.A. (2006). Humor styles, culture-related personality, well-being, and family adjustment among Armenians in Lebanon. Humor: International Journal of Humor Research, 19, 405-423.

Kolstad, A., \& Horpestad, S. (2009). Self-construal in Chile and Norway: Implications for cultural differences in individualism and collectivism. Journal of Cross-cultural Psychology, 40, 275-281.

Kuiper, N.A., Grimshaw, M., Leite, C., \& Kirsh, G. (2004). Humor is not always the best medicine: Specific components of sense of humor and psychological well-being. 
Humor: International Journal of Humor Research, 17, 1351-168.

Kuiper, N. A., Martin, R. A., \& Olinger, L. J. (1993). Coping humor, stress, and cognitive appraisals. Canadian Journal of Behavioural Science, 25, 81-96.

Kuiper, N.A., McKenzie, S. D., \& Belanger, K. A. (1995). Cognitive appraisals and individual differences in sense of humor: Motivational and affective implications. Personality and Individual Differences, 19, 359-372.

Markus, H.R. \& Kitayama, S. (1991). Culture and self: Implications for cognition, emotion, and motivation. Psychological Review, 98, 224-253.

Martin, R. A. (2007). The psychology of humor: An integrative approach. New York: Academic Press.

Martin, R. A., Puhlik-Doris, P., Larsen, G., Gray, J., \& Weir, K. (2003). Individual differences in uses of humor and their relation to psychological well-being: Development of the Humor Styles Questionnaire. Journal of Research in Personality, 37, 48-75.

Saroglou, V., \& Scariot, C. (2002). Humor Styles Questionnaire: Personality and educational correlates in Belgian high school and college students. European Journal of Personality, 16, 43-54.

Singelis, T.M. (1994). The measurement of independent and interdependent selfconstruals. Personality and Social Psychology Bulletin, 20, 580-591.

Taher, D., Kazarian, S.S., \& Martin, R.A. (2008). Validation of the Arabic Humor Styles Questionnaire in a community sample of Lebanese in Lebanon. Journal of Cross-cultural Psychology, 39, 552-564.

Vernon, P.A., Martin, R.A., Schermer, J.A., \& Mackie, A. (2008). A behavioral genetic investigation of humor styles and their correlations with the Big Five personality dimensions. Personality and Individual Differences, 44, 116-1125.

About the authors:

Nicholas A. Kuiper has been a professor in the Department of Psychology at the University of Western Ontario, London, Ontario, Canada since 1978. He has just completed a $2^{\text {nd }}$ term as the Director of the Clinic al Psychology Graduate Program at Western. Over the years he has published numerous research articles on humor, well-being, self-schemata and depression. 
Address for correspondence: Address correspondence to: N. Kuiper, Department of Psychology, Westminster Hall, University of Western Ontario, London, Ontario, Canada N6A 3K7

E-mail: kuiper@uwo.ca

Shahe S. Kazarian has been a professor of clinical psychology at the American University of Beirut since 2001. His research interests focus on cultural clinical psychology, cultural health psychology, family functioning, and the psychology of humor.

Jessica Sine conducted research on humor and interpersonal perceptions with Dr. Kuiper as part of her psychology degree at the Univ ersity of Western Ontario.

Margaret Bassil completed her MA in Psychology at the Americ an University of Beirut in June 2008. Her research interests involve father-child relationships, humor styles, and psychological well-being. She is currently an instructor at the American Univ ersity of Beirut. 